\title{
From multiple integrals to Fredholm determinants
}

\author{
Alexander Seel1, Frank Göhmann² \\ and Andreas Klümper 3 \\ ${ }^{1}$ Institut für Theoretische Physik, Leibniz Universität Hannover, \\ 30167 Hannover, Germany \\ ${ }^{2,3}$ Fachbereich C - Physik, Bergische Universität Wuppertal, \\ 42097 Wuppertal, Germany
}

\begin{abstract}
We show how a multiple integral representation for the density-density correlation functions of the one-dimensional Bose gas with delta function interaction turns into a Fredholm determinant in the limit of infinite repulsion.
\end{abstract}

PACS: 02.30.Ik, 05.30.-d, 05.30.Jp

\footnotetext{
${ }^{1}$ e-mail: alexander.seel@itp.uni-hannover.de

${ }^{2}$ e-mail: goehmann@physik.uni-wuppertal.de

${ }^{3}$ e-mail: kluemper@physik.uni-wuppertal.de
} 


\section{Introduction}

The one-dimensional Bose gas with delta function interaction (contact interaction) is a paradigmatic solvable [14] many-body quantum system. It shares its $R$ matrix underlying the integrability with the spin- $\frac{1}{2}$ Heisenberg chain, but may be considered even simpler, since its Bethe ansatz equations have only real roots [10]. This feature, the absence of strings, in first place simplifies the thermodynamic Bethe ansatz (TBA) analysis, which is the reason why the Bose gas was the first non-trivial Bethe ansatz solvable model whose thermodynamics was analyzed in detail [18].

When in the 80s and early 90s the algebraic Bethe ansatz was applied to the study of correlation functions of solvable models, the Bose gas was again in the center of interest [10]. Explicit results, generalizing early work [12, 13, 15], were obtained in particular for the case of infinite repulsion, the so called impenetrable Boson limit. In this limit many correlation functions can be expressed as Fredholm determinants or Fredholm minors related to integral operators of a special type, the so-called integrable integral operators $[4,10,11]$, which have a close connection with classical integrable evolution equations and for this reason are suitable for an explicit calculation of the asymptotics of correlation functions.

Another type of expression for the correlation functions of solvable many-body systems are the so-called multiple integral representations first obtained in [5] for the density matrix of a segment of the XXZ Heisenberg chain by an approach based on the representation theory of the quantum affine algebra $U_{q}\left(\widehat{\mathfrak{s} l} l_{2}\right)$. Later this result was rederived by means of the algebraic Bethe ansatz [9] which was important, since the algebraic Bethe ansatz turned out to be flexible enough for a number of generalizations. In our context we would like to mention the works [8] and [2], where multiple integral representations for a one-parameter generating function of the $z z$-correlation functions of the XXZ chain were derived for the ground state and for finite temperature. From [8] it was only a small step to the corresponding expression for the Bose gas [6], namely a multiple integral representation for a generating function of the density-density correlation functions. A finite temperature version was obtained directly from [2] in a certain scaling limit close to the ferromagnetic point $\Delta=-1$ of the XXZ chain [16].

The latter is the starting point of this work and is reviewed in the next section. Then, in section 3, we perform the impenetrable Boson limit and show that the resulting expression is equal to a known Fredholm determinant representation. This is the main result we wish to convey: in an appropriate limit the multiple integrals turn into a Fredholm determinant. Looking at it from a different angle this means, that we may interpret the multiple integrals as a 'deformation of a Fredholm determinant'. To round off this work we recall how to obtain the density-density correlation function of the Bose gas from the Fredholm determinant in section 4. Section 5 is devoted to a concluding summary. 


\section{Density-Density Correlations in the Bose Gas}

The one-dimensional Bose gas with contact interaction is described by the Hamiltonian

$$
H=\int_{0}^{\ell} \mathrm{d} z\left[\left(\partial_{z} \psi^{\dagger}\right)\left(\partial_{z} \psi\right)+c \psi^{\dagger}(z) \psi^{\dagger}(z) \psi(z) \psi(z)\right] .
$$

Here $\psi^{\dagger}(z)$ and $\psi(z)$ are Bose fields with canonical equal-time commutation relations which act on the interval $[0, \ell]$ for which we assume periodic boundary conditions. $c>0$ is the coupling constant.

The operator

$$
Q(x)=\int_{0}^{x} \mathrm{~d} z \psi^{\dagger}(z) \psi(z)
$$

measures the number of particles in the interval $[0, x], 0 \leq x \leq \ell$. We need it to define a one-parameter generating function of the density-density correlation functions by $\left\langle\mathrm{e}^{\varphi Q(x)}\right\rangle_{T, \mu}$, where the brackets indicate the grand-canonical ensemble average for a heat and particle bath of temperature $T$ and with chemical potential $\mu$. The function $\left\langle\mathrm{e}^{\varphi Q(x)}\right\rangle_{T, \mu}$ is particularly convenient in the context of the algebraic Bethe ansatz [10]. With the shorthand notation $j(x)=\psi^{\dagger}(x) \psi(x)$ for the particle density operator we have the following formulae,

$$
\langle j(x)\rangle_{T, \mu}=\left.\partial_{x} \partial_{\varphi}\left\langle\mathrm{e}^{\varphi Q(x)}\right\rangle_{T, \mu}\right|_{\varphi=0}, \quad\langle j(0) j(x)\rangle_{T, \mu}=\left.\frac{1}{2} \partial_{x}^{2} \partial_{\varphi}^{2}\left\langle\mathrm{e}^{\varphi Q(x)}\right\rangle_{T, \mu}\right|_{\varphi=0} .
$$

In our previous work [16] we obtained the multiple integral representation

$$
\begin{aligned}
\left\langle\mathrm{e}^{\varphi Q(x)}\right\rangle_{T, \mu}= & \sum_{n=0}^{\infty} \frac{1}{(n !)^{2}}\left[\prod_{j=1}^{n} \int_{\mathbb{R}} \frac{\mathrm{d} p_{j}}{2 \pi} \frac{\mathrm{e}^{\mathrm{i} p_{j} x}}{1+\mathrm{e}^{\varepsilon\left(p_{j}\right) / T}} \int_{\mathbb{R}+\mathrm{i} 0} \frac{\mathrm{d} q_{j}}{2 \pi} \mathrm{e}^{-\mathrm{i} q_{j} x}\right] \\
& {\left[\prod_{j, k=1}^{n} \frac{p_{j}-q_{k}-\mathrm{i} c}{q_{j}-q_{k}-\mathrm{i} c}\right] \operatorname{det}\left[M\left(p_{j}, q_{k}\right)\right]_{j, k=1, \ldots n} \operatorname{det}\left[G\left(p_{j}, q_{k}\right)\right]_{j, k=1, \ldots n} }
\end{aligned}
$$

for the generating function in the thermodynamic limit by considering a special scaling limit of the XXZ chain close to the isotropic ferromagnetic point $\Delta=-1$. Here $M(p, q)$ is defined as 1

$$
M(p, q)=\frac{\mathrm{i}}{(p-q)}\left[\frac{\mathrm{i} c}{p-q+\mathrm{i} c} \prod_{l=1}^{n} \frac{p-q_{l}+\mathrm{i} c}{p-p_{l}+\mathrm{i} c}+\frac{\mathrm{i} c \mathrm{e}^{\varphi}}{p-q-\mathrm{i} c} \prod_{l=1}^{n} \frac{p-q_{l}-\mathrm{i} c}{p-p_{l}-\mathrm{i} c}\right] .
$$

The temperature and the chemical potential enter through the functions $\varepsilon(p)$ and $G(p, q)$ which must be calculated as solutions of integral equations. The

\footnotetext{
${ }^{1}$ Note a typo in equation (26) of the published version of [16]: the first factor of the second term on the rhs should read $c \mathrm{e}^{\varphi} /\left(w_{j}-p_{k}\right)\left(w_{j}-p_{k}-\mathrm{i} c\right)$ instead of $c \mathrm{e}^{\varphi} /\left(w_{j}-p_{k}\right)\left(w_{j}-p_{k}+\mathrm{i} c\right)$.
} 
'dressed energy function' $\varepsilon(p)$ is the solution of the non-linear integral equation

$$
\varepsilon(p)=p^{2}-\mu-T \int_{\mathbb{R}} \frac{\mathrm{d} q}{\pi} \frac{c}{(p-q)^{2}+c^{2}} \ln \left(1+\mathrm{e}^{-\varepsilon(q) / T}\right)
$$

of Yang and Yang [18]. The 'density function' $G(p, q)$ solves the linear integral equation

$$
G(p, q)=-\frac{c}{(p-q)(p-q-\mathrm{i} c)}+\int_{\mathbb{R}} \frac{\mathrm{d} k}{\pi} \frac{c}{(p-k)^{2}+c^{2}} \frac{G(k, q)}{1+\mathrm{e}^{\varepsilon(k) / T}} .
$$

\section{Impenetrable Boson Limit}

It is known since the work of Girardeau [1] that in the limit $c \rightarrow \infty$ the wave functions of Bosons with contact interaction turn into those of free Fermions, up to a function which takes values \pm 1 . We therefore expect the multiple integral formula (41) to simplify in this limit. In the following we indicate the limit by supplying a superscript (0) to the respective functions.

We first of all note that the kernel in the integral equations (66) and (77) for $\varepsilon(p)$ and $G(p, q)$ vanishes for $c \rightarrow \infty$, such that their solutions become explicit,

$$
\varepsilon^{(0)}(p)=p^{2}-\mu, \quad G^{(0)}(p, q)=\frac{-\mathrm{i}}{p-q} .
$$

Consequently the expression $1 /\left(1+\mathrm{e}^{\varepsilon(p) / T}\right)$ turns into the Fermi function

$$
\vartheta(p)=\frac{1}{1+\mathrm{e}^{\left(p^{2}-\mu\right) / T}}
$$

for non-relativistic particles. Moreover, the function $M(p, q)$ simplifies drastically for $c \rightarrow \infty$,

$$
M^{(0)}(p, q)=\frac{\mathrm{i}\left(1-\mathrm{e}^{\varphi}\right)}{p-q}
$$

and the explicit product of the right hand side of equation (44) converges to one.

Substituting (8) and (10) into the determinants in (41) we see that they are of Cauchy-type in the limit. Using the well-known formula

$$
\operatorname{det}\left(\frac{1}{p_{j}-q_{k}}\right)_{j, k=1, \ldots, n}=\frac{\prod_{a<b}\left(p_{a}-p_{b}\right)\left(q_{b}-q_{a}\right)}{\prod_{a, b=1}^{n}\left(p_{a}-q_{b}\right)}
$$

we obtain

$$
\begin{aligned}
& \left\langle\mathrm{e}^{\varphi Q(x)}\right\rangle_{T, \mu}^{(0)}= \\
& \quad \sum_{n=0}^{\infty} \frac{\left(1-\mathrm{e}^{\varphi}\right)^{n}}{(n !)^{2}}\left[\prod_{j=1}^{n} \int_{\mathbb{R}} \frac{\mathrm{d} p_{j}}{2 \pi} \mathrm{e}^{\mathrm{i} p_{j} x} \vartheta\left(p_{j}\right) \int_{\mathbb{R}+\mathrm{i} 0} \frac{\mathrm{d} q_{j}}{2 \pi} \mathrm{e}^{-\mathrm{i} q_{j} x}\right] \frac{\Delta^{2}(p) \Delta^{2}(q)}{\prod_{j, k=1}^{n}\left(q_{j}-p_{k}\right)^{2}},
\end{aligned}
$$


where we introduced the notation

$$
\Delta(p)=\prod_{j<k}\left(p_{k}-p_{j}\right)=\operatorname{det}\left(p_{k}^{j-1}\right)_{j, k=1, \ldots, n}
$$

for Vandermonde determinants.

Due to the symmetry of the integrand with respect to all $q_{j}$, one of the Vandermonde determinants $\Delta(q)$ in each term can be replaced by a product of the diagonal elements of the Vandermonde matrix and subsequently the $q$-integrals can be pulled into the second Vandermonde determinant 2 Then

$$
\frac{1}{n !}\left[\prod_{l=1}^{n} \int_{\mathbb{R}+\mathrm{i} 0} \frac{\mathrm{d} q_{l}}{2 \pi} \mathrm{e}^{-\mathrm{i} q_{l} x}\right] \frac{\Delta^{2}(q)}{\prod_{j, k=1}^{n}\left(q_{j}-p_{k}\right)^{2}}=\operatorname{det}\left[\int_{\mathbb{R}+\mathrm{i} 0} \frac{\mathrm{d} q}{2 \pi} \frac{\mathrm{e}^{-\mathrm{i} q x} q^{j+k-2}}{\prod_{l=1}^{n}\left(q-p_{l}\right)^{2}}\right]_{j, k=1, \ldots, n}
$$

Obviously the integrals inside the determinant on the right hand side can now be calculated by means of the residue theorem. Yet, it turns out to be more convenient to perform a number of elementary row- and column operations first, resulting in the sequence of identities

$$
\begin{aligned}
\operatorname{det}\left[\int_{\mathbb{R}+\mathrm{i} 0} \frac{\mathrm{d} q}{2 \pi}\right. & \left.\frac{\mathrm{e}^{-\mathrm{i} q x} q^{j+k-2}}{\prod_{l=1}^{n}\left(q-p_{l}\right)^{2}}\right]_{j, k=1, \ldots, n} \\
& =\operatorname{det}\left[\int_{\mathbb{R}+\mathrm{i} 0} \frac{\mathrm{d} q}{2 \pi} \frac{\mathrm{e}^{-\mathrm{i} q x} \prod_{a=1}^{j-1}\left(q-p_{a}\right) \prod_{b=1}^{k-1}\left(q-p_{b}\right)}{\prod_{l=1}^{n}\left(q-p_{l}\right)^{2}}\right]_{j, k=1, \ldots, n} \\
& =\frac{1}{\Delta^{2}(p)} \operatorname{det}\left[\int_{\mathbb{R}+\mathrm{i} 0} \frac{\mathrm{d} q}{2 \pi} \frac{\mathrm{e}^{-\mathrm{i} q x}}{\left(q-p_{j}\right)\left(q-p_{k}\right)}\right]_{j, k=1, \ldots, n} \\
& =\frac{1}{\Delta^{2}(p)} \operatorname{det}\left[-\frac{2 \sin \left(\frac{p_{j}-p_{k}}{2} x\right)}{p_{j}-p_{k}} \mathrm{e}^{-\mathrm{i}\left(p_{j}+p_{k}\right) x / 2}\right]_{j, k=1, \ldots, n} .
\end{aligned}
$$

In the last line for $j=k$ it is understood to take the analytic continuation of the function

$$
V(u, v)=\frac{2 \sin \left(\frac{u-v}{2} x\right)}{u-v},
$$

namely $V(u, u)=x$, which comes from the calculation of the residua at the second order poles of the diagonal entries. Finally pulling out the exponential factors and a minus sign in (15) we end up with the expression

$$
\operatorname{det}\left[\int_{\mathbb{R}+\mathrm{i} 0} \frac{\mathrm{d} q}{2 \pi} \frac{\mathrm{e}^{-\mathrm{i} q x} q^{j+k-2}}{\prod_{l=1}^{n}\left(q-p_{l}\right)^{2}}\right]_{j, k=1, \ldots, n}=\frac{\mathrm{e}^{-\mathrm{i} \sum_{j=1}^{n} p_{j} x}}{(-1)^{n} \Delta^{2}(p)} \operatorname{det}\left[V\left(p_{j}, p_{k}\right)\right]_{j, k=1, \ldots, n} .
$$

\footnotetext{
${ }^{2}$ The same trick was used for the free Fermion limit of the XXZ chain in [7].
} 
We further introduce the notation

$$
V_{T}(u, v)=\sqrt{\vartheta(u)} V(u, v) \sqrt{\vartheta(v)}
$$

and substitute it together with (14) and (17) into (12) to obtain

$$
\left\langle\mathrm{e}^{\varphi Q(x)}\right\rangle_{T, \mu}^{(0)}=\sum_{n=0}^{\infty} \frac{\left(\mathrm{e}^{\varphi}-1\right)^{n}}{n !}\left[\prod_{j=1}^{n} \int_{\mathbb{R}} \frac{\mathrm{d} p_{j}}{2 \pi}\right] \operatorname{det}\left[V_{T}\left(p_{j}, p_{k}\right)\right]_{j, k=1, \ldots, n} .
$$

Now recall (see e.g. [17]) that for an integral operator $\widehat{K}$ with kernel $K(p, q)$ acting on a function $\phi$ defined on an interval $I$ as

$$
(\widehat{K} \phi)(p)=\int_{I} \mathrm{~d} q K(p, q) \phi(q)
$$

its Fredholm determinant has the infinite series representation

$$
\operatorname{det}(1+\widehat{K})=\sum_{n=0}^{\infty} \frac{1}{n !} \int_{I^{n}} \mathrm{~d} p_{1} \ldots \mathrm{d} p_{n} \operatorname{det}\left[K\left(p_{j}, p_{k}\right)\right]_{j, k=1, \ldots, n}
$$

Then

$$
\left\langle\mathrm{e}^{\varphi Q(x)}\right\rangle_{T, \mu}^{(0)}=\operatorname{det}\left(1+\frac{\mathrm{e}^{\varphi}-1}{2 \pi} \widehat{V}_{T}\right),
$$

where $\widehat{V}_{T}$ is the integral operator with kernel $V_{T}(p, q)$. This is a known result [10]. What we find remarkable, however, is that it directly follows from the multiple integral representation (41).

\section{One- and Two-Point Functions}

In this section we would like to recall briefly how to obtain the one- and twopoint density correlation functions by applying (3) to (19). Because $\varphi$ appears in (19) only in the factor $\left(\mathrm{e}^{\varphi}-1\right)^{n}$, the derivatives with respect to $\varphi$ can be easily calculated by means of the formula

$$
\left.\partial_{\varphi}\left(\mathrm{e}^{\varphi}-1\right)^{n}\right|_{\varphi=0}=\delta_{n, 1},\left.\quad \partial_{\varphi}^{2}\left(\mathrm{e}^{\varphi}-1\right)^{n}\right|_{\varphi=0}=\delta_{n, 1}+2 \delta_{n, 2} .
$$

Then we obtain the known [10] explicit expression

$$
\langle j(x)\rangle_{T, \mu}^{(0)}=\partial_{x} \int_{\mathbb{R}} \frac{\mathrm{d} p}{2 \pi} \vartheta(p) x=\int_{\mathbb{R}} \frac{\mathrm{d} p}{2 \pi} \vartheta(p)=D(T, \mu),
$$

which due to translational invariance is independent of the interval length $x$. 
As the term arising from $n=1$ is linear in $x$ we need to consider only the $n=2$ term in order to calculate the density-density correlation function,

$$
\begin{aligned}
2 \frac{\partial^{2}}{\partial x^{2}} \frac{1}{2 !} \int_{\mathbb{R}} \frac{\mathrm{d} p_{1}}{2 \pi} \int_{\mathbb{R}} \frac{\mathrm{d} p_{2}}{2 \pi} \operatorname{det}\left[V_{T}\left(p_{j}, p_{k}\right)\right]_{j, k=1,2} \\
\quad=\frac{\partial^{2}}{\partial x^{2}} \int_{\mathbb{R}} \frac{\mathrm{d} p_{1}}{2 \pi} \int_{\mathbb{R}} \frac{\mathrm{d} p_{2}}{2 \pi} \vartheta\left(p_{1}\right) \vartheta\left(p_{2}\right)\left[x^{2}-\frac{4 \sin ^{2}\left(\frac{p_{1}-p_{2}}{2} x\right)}{\left(p_{1}-p_{2}\right)^{2}}\right] \\
=2\left[\int_{\mathbb{R}} \frac{\mathrm{d} p}{2 \pi} \vartheta(p)\right]^{2}-2 \int_{\mathbb{R}} \frac{\mathrm{d} p_{1}}{2 \pi} \int_{\mathbb{R}} \frac{\mathrm{d} p_{2}}{2 \pi} \vartheta\left(p_{1}\right) \vartheta\left(p_{2}\right) \cos \left(p_{1} x-p_{2} x\right) \\
=2 D^{2}(T, \mu)-2\left[\int_{\mathbb{R}} \frac{\mathrm{d} p}{2 \pi} \vartheta(p) \cos (p x)\right]^{2} .
\end{aligned}
$$

Hence, we have arrived at another known result [10],

$$
\langle j(0) j(x)\rangle_{T, \mu}^{(0)}-D^{2}(T, \mu)=-\left[\int_{\mathbb{R}} \frac{\mathrm{d} p}{2 \pi} \vartheta(p) \mathrm{e}^{\mathrm{i} p x}\right]^{2} .
$$

\section{Conclusion}

In the limit of impenetrable Bosons we transformed a multiple integral representation for the generating function of the density-density correlation functions of the Bose gas with contact interaction into a Fredholm determinant of an integrable integral operator. The latter provides a link to classical integrable evolution equations and thus a means for a rigorous analysis of the asymptotics of correlation functions [3]. Taking this into account, it is tempting to interpret the multiple integral representation as a 'deformation of a Fredholm determinant'. One may hope that such an interpretation will finally help to solve the problem of the calculation of the long-distance asymptotics also in the generic case of finite repulsion.

Acknowledgement. AS gratefully acknowledges financial support by the German Science Foundation under grant number Se 1742 1-1.

\section{References}

[1] M. Girardeau, Relationship between systems of impenetrable Bosons and Fermions in one dimension, J. Math. Phys. 1 (1960), 516.

[2] F. Göhmann, A. Klümper, and A. Seel, Integral representations for correlation functions of the XXZ chain at finite temperature, J. Phys. A: Math. Gen. 37 (2004), 7625. 
[3] A. R. Its, A. G. Izergin, and V. E. Korepin, Long-distance asymptotics of temperature correlators of the impenetrable Bose gas, Comm. Math. Phys. 130 (1990), 471.

[4] - Temperature correlators of the impenetrable Bose gas as an integrable system, Comm. Math. Phys. 129 (1990), 205.

[5] M. Jimbo, K. Miki, T. Miwa, and A. Nakayashiki, Correlation functions of the XXZ model for $\Delta<-1$, Phys. Lett. A 168 (1992), 256.

[6] N. Kitanine, K. Kozlowski, J. M. Maillet, N. A. Slavnov, and V. Terras, On correlation functions of integrable models associated with the six-vertex R-matrix, J. Stat. Mech. (2007), P01022.

[7] N. Kitanine, J. M. Maillet, N. A. Slavnov, and V. Terras, Correlation functions of the XXZ spin- $\frac{1}{2}$ Heisenberg chain at the free fermion point from their multiple integral representations, Nucl. Phys. B 642 (2002), 433.

[8] Spin-spin correlation functions of the XXZ- $\frac{1}{2}$ Heisenberg chain in a magnetic field, Nucl. Phys. B 641 (2002), 487.

[9] N. Kitanine, J. M. Maillet, and V. Terras, Correlation functions of the XXZ Heisenberg spin- $\frac{1}{2}$ chain in a magnetic field, Nucl. Phys. B 567 (2000), 554.

[10] V. E. Korepin, N. M. Bogoliubov, and A. G. Izergin, Quantum Inverse Scattering Method and Correlation Functions, Cambridge University Press, 1993.

[11] V. E. Korepin and N. A. Slavnov, The time-dependent correlation function of an impenetrable Bose gas as a Fredholm minor, Comm. Math. Phys. 129 (1990), 103.

[12] A. Lenard, Momentum distribution in the ground state of the onedimensional system of impenetrable Bosons, J. Math. Phys. 5 (1964), 930.

[13] _ One-dimensional impenetrable Bosons in thermal equilibrium, J. Math. Phys. 7 (1966), 1268.

[14] E. H. Lieb and W. Liniger, Exact analysis of an interacting Bose gas. I. The general solution and the ground state, Phys. Rev. 130 (1963), 1605.

[15] T. D. Schultz, Note on the one-dimensional gas of impenetrable point-particle Bosons, J. Math. Phys. 4 (1963), 666.

[16] A. Seel, T. Bhattacharyya, F. Göhmann, and A. Klümper, A note on the spin- $\frac{1}{2}$ XXZ chain concerning its relation to the Bose gas, J. Stat. Mech. (2007), P08030. 
[17] E. T. Whittaker and G. N. Watson, A Course of Modern Analysis, fourth ed., ch. 11, Cambridge University Press, 1963.

[18] C. N. Yang and C. P. Yang, Thermodynamics of a one-dimensional system of Bosons with repulsive delta-function interaction, J. Math. Phys. 10 (1969), 1115. 